\title{
7 ADHS und Sucht
}

\author{
Frank Häßler
}

\subsection{Einleitung}

Hyperkinetische Störungen (ICD-10) zählen mit einer Prävalenz von 2-6\% zu den häufigsten kinder- und jugendpsychiatrischen Störungen, deren Symptome in 30 bis 50 \% aller Fälle bis in das Erwachsenenalter persistieren. Im Folgenden werden HKS und Aufmerksamkeits-/Hyperaktivitätsstörung (ADHS) synonym verwandt.

HKS sind im Kindes- und Jugendalter durch ein situationsübergreifendes Muster von Unaufmerksamkeit, Überaktivität und Impulsivität, welches für den Entwicklungsstand des Betroffenen ein abnormes Ausmaß erreicht, gekennzeichnet. Im Erwachsenenalter stehen eher Probleme exekutiver Funktionen, der Aufmerksamkeitsfokussierung, der emotionalen Modulation, der Alltagstrukturierung und der Impulskontrolle im Vordergrund. Mit zunehmendem Alter sind HKS bis zu 90\% mit einer oder mehreren anderen psychischen Störungen assoziiert. Neben einer Heredität von ca. 80 \% weisen nicht nur molekulargenetische, sondern auch funktionelle und bildgebende Untersuchungen auf eine Störung des Stoffwechsels/der Balance der Neurotransmitter Dopamin und Noradrenalin in bestimmten Hirnregionen (limbisches System und Stirnhirnbereich) hin. Neuropsychologische Konzepte gehen davon aus, dass den HKS exekutive Dysfunktionen in den Bereichen Aufmerksamkeit, Impulskontrolle einschließlich Vorbereitung, Auswahl und Durchführung motorischer Abläufe, Arbeitsgedächtnis, Verzögerungsaversion und Zeitdiskrimination zugrunde liegen. Die Behandlung sollte multimodal erfolgen und umfasst in Abhängigkeit vom Alter des Betroffenen neben der Psychopharmakotherapie mit Stimulanzien (Methylphenidat in unretardierter oder retardierter Formulierung) oder Atomoxetin vor allem Psychoedukation, 
Elterntraining, Familientherapie und kognitiv behaviorale Therapien. Komorbide Störungen bedürfen ergänzender leitlinienkonformer Therapien.

\subsection{Begriffsbestimmungen}

\section{Schädlicher Gebrauch (ICD-10: F1x.1) = Missbrauch}

Von schädlichem Gebrauch spricht man, wenn das Konsumverhalten zu psychischen, sozialen und/oder organischen Schäden führt. Die Palette reicht dabei von depressiven dysthymen Symptomen über neurotoxische, z. B. Polyneuropathien, Enzephalopathien, zerebrale Krampfanfälle, bis hin zum sozialen Abstieg (Arbeitsplatzverlust etc.).

\section{Abhängigkeitssyndrom}

Abhängigkeit ist kein einheitliches Erscheinungsbild (Uchtenhagen 2000). Die frühere Differenzierung in körperliche und psychische Abhängigkeit ist aufgrund der erschwerten und unscharfen klinischen Unterscheidung aufgegeben worden. In den unterschiedlichen Diagnosesystemen ICD-10 und DSM-IV sind entweder 8 oder 9 Kriterien enthalten, von denen 3 mindestens einen Monat oder mehrfach in den vergangenen 12 Monaten bestanden haben müssen.

Diagnosekriterien für das Abhängigkeitssyndrom gemäß ICD-10 und DSM-IV:

- starker Wunsch oder eine Art Zwang, Substanzen oder Alkohol zu konsumieren

v verminderte Kontrollfähigkeit bezüglich des Beginns, der Beendigung und der Menge des Substanz- oder Alkoholkonsums

- Substanzgebrauch mit dem Ziel, Entzugssymptome zu mildern und der entsprechenden positiven Erfahrung

- körperliches Entzugssyndrom

- Nachweis einer Toleranz; um die ursprünglich durch niedrige Dosen erreichten Wirkungen der Substanz hervorzurufen, sind zunehmend höhere Dosen erforderlich (eindeutige Beispiele hierfür sind die Tagesdosen von Alkoholikern und Opiatabhängigen, die Konsumenten ohne Toleranzentwicklung schwer beeinträchtigen würden oder bei diesen sogar zum Tode führen würden)

- Ein eingeengtes Verhaltensmuster im Umgang mit Alkohol oder der Substanz wie z. B. die Tendenz, Alkohol an den Werktagen wie an Wochenenden $\mathrm{zu}$ trinken und die Regeln eines gesellschaftlich üblichen Trinkverhaltens außer Acht zu lassen

- Fortschreitende Vernachlässigung anderer Vergnügungen oder Interessen zugunsten des Substanzkonsums 
- Anhaltender Substanz- oder Alkoholkonsum trotz Nachweis eindeutiger schädlicher Folgen; die schädlichen Folgen können körperlicher Art sein, wie z.B. Leberschädigung durch exzessives Trinken oder sozial, wie Arbeitsplatzverlust durch eine substanzbedingte Leistungseinbuße, oder psychisch, wie bei depressiven Zuständen nach massivem Substanzkonsum

Im DSM-IV wird der „starke Wunsch“, eine Substanz zu konsumieren, nicht erwähnt. Hingegen findet ein weiteres Kriterium Berücksichtigung: anhaltender Wunsch oder erfolglose Versuche, den Substanzgebrauch zu verringern oder zu kontrollieren.

\subsection{Komorbiditäten}

Komorbide Störungen sind sowohl bei Kindern und Jugendlichen als auch Erwachsenen mit ADHS weit verbreitet. Nicht wenige entstehen sekundär durch die ADHS-Symptomatik (Barkley 2006). Die Frage ist, ob es sich um Koinzidenzen oder um primäre bzw. sekundäre Komorbiditäten handelt. Während ältere Studien noch von der Annahme ausgingen, dass beim Mischtyp höhere Komorbiditätsraten auftreten, fanden Sprafkin et al. (2007), dass alle drei Subtypen mit hohen Komorbiditätsraten einhergehen, die schwersten Ausprägungen aber beim hyperaktiv-impulsiven, unaufmerksamen Mischtyp vorkommen (s. Tab. 4).

Tab. 4 Komorbiditäten bei Kindern und Erwachsenen mit ADHS (Gillberg et al. 2004; Sobanski 2006, Pliszka et al. 2007); * Häufigkeit von ADHS unter Patienten mit den erwähnten psychischen Störungen; SSV = Störung des Sozialverhaltens; AS = Angststörung; PS = Persönlichkeitsstörung

\begin{tabular}{|c|c|c|}
\hline komorbide Störung & Prävalenz Kinder & Prävalenz Erwachsene \\
\hline Oppositionelle SSV & $50 \%$ & \\
\hline SSV & $30-50 \%$ & \\
\hline $\begin{array}{l}\text { affektive (depressive) } \\
\text { Störungen }\end{array}$ & $10-40 \%$ & 35-50\% (lifetime) \\
\hline affektive (manische) Störungen & $16 \%$ & \\
\hline Bipolare Störungen & $60-90 \%$ & $9,5 \% *$ \\
\hline Angststörungen & $20-30 \%$ & $40-60 \%$ (lifetime) \\
\hline soziale Phobie & & 20-34\% (lifetime) \\
\hline generalisierte AS & & $10-45 \%$ \\
\hline Panikstörung & & $9,5 \% *$ \\
\hline Teilleistungsstörungen & $10-40 \%$ & \\
\hline Lese-Rechtschreib-Schwäche & ca. $25 \%$ & \\
\hline
\end{tabular}




\begin{tabular}{lcc}
\hline komorbide Störung & Prävalenz Kinder & Prävalenz Erwachsene \\
\hline Einschlafstörungen & ca. $50 \%$ & ca. $70 \%$ \\
\hline $\begin{array}{l}\text { Somatisierungsstörungen } \\
\text { Ticstörungen }\end{array}$ & $20-25 \%$ & \\
\hline $\begin{array}{l}\text { Alkoholmissbrauch/ } \\
\text { Abhängigkeit }\end{array}$ & $30-50 \%$ & $25-52 \%$ \\
\hline $\begin{array}{l}\text { Drogenmissbrauch } \\
\text { Nikotinmissbrauch/ }\end{array}$ & $17-45 \%$ & $25-35 \%$ \\
\hline -abhängigkeit & $9-30 \%$ & $30-38 \%$ \\
\hline Bulimia nervosa & $15-19 \%$ & $40 \%$ \\
\hline antisoziale PS & $8 \%$ & $3-9 \%$ \\
\hline Autismus & $6 \%$ & $18-23 \%$ \\
\hline Epilepsie & & $3,5 \%$ \\
\hline
\end{tabular}

Erwachsene mit ADHS vs. nicht ADHS haben bzgl. einer lifetime Psychopathologie in $87 \%$ vs. $64 \%$ mindestens eine und in 56 vs. $27 \%$ zwei psychiatrische Störungen. Das Morbiditätsrisiko beträgt 16,5 vs. 3,9\% für eine Störung des Sozialverhaltens, 72,7 vs. 8,6\% für Major Depression, 17,5 vs. 8,3\% für Angststörungen, 56,6 vs. 32,9\% für Substanzmissbrauch und 28,o vs . 1,1\% für oppositionell abweichendes Verhalten (McGough et al. 2006).

\section{$7.4 \quad$ Nikotin}

Nikotin ist ein Alkaloid, das vorwiegend in der Tabakpflanze (5\% Massenanteil), insbesondere in den Blättern vorkommt. Nikotin depolarisiert die postsynaptische Membran und wirkt in geringeren Dosen ähnlich ganglienerregend wie Azetylcholin, in höherer Konzentration und bei längerer Depolarisation dann blockierend. Neuronale nikotinische Azetylcholinrezeptoren bestehen aus bisher 8 nachgewiesenen Untereinheiten, wobei desensibilisierte Rezeptoren eine höhere Affinität für Nikotin als aktivierte haben. Aus der Aktivierung verschiedener Neurotransmitter, bedingt durch die akute Depolarisation der Nervenendigungen, erklärt sich die komplexe vielgestaltige Wirkung. Gedächtnissteigerung und Vasokonstriktion der Hautgefäße lassen sich am ehesten mit der gesteigerten Vasopressinfreisetzung erklären. Die Katecholamine Noradrenalin und Adrenalin sind für Anstieg der Herzfrequenz, des Blutdrucks, der Konstriktion der Koronargefäße und der myokardialen Kontraktilität verantwortlich. Serotonin hemmt einerseits den Appetit, verbessert andererseits aber die Stimmung. Das Ansprechen des mesolimbischen Dopaminsystems stellt eine Aktivierung des belohnenden Systems dar. Es kommt zu einer kurzzeitigen Steigerung der psychomotorischen Leistungsfähigkeit und 
der Aufmerksamkeit. Über $\beta$-Endorphine wird eine Angst- und Stressminderung bewirkt (Domino 1998).

Aufgrund dieser Eigenschaften entfaltet Nikotin eine ähnliche Wirkung wie die Stimulanzien, nur mit dem Unterschied, dass Nikotin ein hohes Suchtpotenzial besitzt. Nikotin steht in einem vielfältigen Zusammenhang zur ADHS. Einerseits korreliert exzessiver mütterlicher Nikotinkonsum während der Schwangerschaft signifikant mit der späteren Manifestation einer HKS bei dem betroffenen Kind (Schulze und Trott 1996; Mick et al. 2002) und andererseits ist insbesondere der hyperaktiv-impulsiv prädominante Typ mit jeglicher Substanzabhängigkeit assoziiert, während der rein unaufmerksame Typ nur mit einer Nikotinabhängigkeit einhergeht (Elkins et al. 2007). Unabhängig von einer komorbiden Störung des Sozialverhaltens kann ADHD die Wahrscheinlichkeit für das Zigarettenrauchen und sogar für eine Nikotinabhängigkeit signifikant erhöhen. In amerikanischen Untersuchungen lag der Raucheranteil bei ADHS-Betroffenen mit $40 \%$ deutlich höher als in der Normalbevölkerung mit $26 \%$ (Sullivan und Rudnik-Levin 2001). Bei Alkoholabhängigen liegt der Raucheranteil bei ca. 70\%, bei Alkoholabhängigen mit ADHS dagegen bei $90 \%$ (Krause et al. 2002).

\subsection{Alkohol}

Ethanol mit der Formel $\mathrm{C}_{2} \mathrm{H}_{5} \mathrm{OH}$ gehört zu den n-Alkanolen. Es leitet sich von dem Alkan (gesättigten Kohlenwasserstoff) Ethan $\left(\mathrm{C}_{2} \mathrm{H}_{6}\right)$ ab, in dem formal ein Wasserstoffatom durch die funktionelle Hydroxylgruppe $(\mathrm{OH})$ ersetzt ist. Deshalb wird an den Namen Ethan das Suffix -ol angehängt. Alkohol (Äthylalkohol) wird im Magen-Darm-Trakt absorbiert, teils schon in der Magenschleimhaut metabolisiert und erreicht nach rund $\mathrm{l} \mathrm{h}$ seine höchste Konzentration im Blut (Blutalkoholkonzentration; BAK). Nicht vollständig metabolisierter Alkohol wird unverändert über die Niere oder die Lunge eliminiert. Das im menschlichen Körper weit verbreitete Enzym Alkoholdehydrogenase (ADH) wandelt hauptsächlich Alkohol zu Azetaldehyd um. Rechtsmediziner gehen von einer Metabolisierungsrate von o,15 Promille/h aus. Azetaldehyd wird dann weiter durch die Aldehyddehydrogenase (AIDH) zu Essigsäure verstoffwechselt und in den Zitronensäurezyklus eingespeist. Alkohol in niedrigen Konzentrationen hat selektive Wirkungen auf neuronale Mechanismen. So verstärkt er die hemmende Wirkung von GABA, wirkt hemmend auf glutamaterge synaptische Übertragungsvorgänge, reduziert akut von NMDA (N-Methyl-D-Aspartat) vermittelte Ionenströme in der postsynaptischen Membran, erhöht bei chronischem Missbrauch die Anzahl der Bindungsstellen für unterschiedliche NMDA-Rezeptorliganden, verändert deren pharmakologische Sensitivität und beeinflusst darüber hinaus die spannungsabhängigen Kalziumkanäle vom L-Typ sowie 5-HT-Rezeptoren (Rommelspacher 2000).

Von der Konstitution, den Trinkgewohnheiten und natürlich der konsumierten Alkoholmenge hängt die körperliche und psychische Symptomatik 
der akuten Intoxikation ab. Im Vordergrund stehen Dysregulationen der Hemmmechanismen, Stimmungslabilität, Suggestibilität, Minderung der Einsichts- und Kritikfähigkeit, Denkverlangsamung, rigidere Informationsverarbeitung, Koordinationsstörungen bis hin zur Veränderung des Sprachflusses, Bewusstseinsveränderung, Tachykardie, arterieller Hypertonus und oberflächliche Gefäßdilatation. In letaler Dosierung, die umso geringer sein kann, je jünger der Intoxikierte ist, kommt es zum Bewusstseinsverlust und zur Atemlähmung. Allgemein bekannte Nachwehen („Kater“) sind Kopfschmerzen, Übelkeit, vegetative Labilität, raschere Erschöpfbarkeit und erhöhte Erregbarkeit durch Sinnesreize.

ADHS-Betroffene scheinen keine spezifischen abhängigkeitserzeugenden Substanzen zu bevorzugen, entwickeln aber besonders früh einen Tabak- und Alkoholkonsum (Kuperman et al. 2001). Bei Alkoholabhängigen wurde bei 25-30 \% ein ADHS bzw. ADHS im Kindes- und Jugendalter beobachtet (Molina und Pelham 2003). Auch bei jungen Erwachsenen mit ADHS bzw. ADHS im Kindesalter trat bei ca. $35 \%$ ein Alkoholmissbrauch bzw. eine Alkoholabhängigkeit im Vergleich zu 6\% in der Kontrollgruppe ohne ADHS auf (Murphy et al. 2002). Um zu ermitteln, ob alkoholabhängige Erwachsene mit komorbider ADHS klinische Besonderheiten aufweisen, untersuchten Wodarz et al. (2004) 314 konsekutiv behandelte alkoholabhängige Patienten (Durchschnittsalter 43 Jahre, $17 \%$ weibliche Patienten). $21,3 \%$ der Patienten (22\% der Männer, $17 \%$ der Frauen) mit einer Alkoholabhängigkeit wiesen eine ADHS im Erwachsenenalter auf. Bei dieser Subgruppe war eine Alkoholabhängigkeit 6 Jahre eher aufgetreten. Auch die tägliche Trinkmenge war um ca. $30 \%$ höher. Soziale Belastungen und Konfrontationen mit der Justiz traten 5 -mal häufiger auf als in der Gruppe der Alkoholabhängigen ohne ADHS. Die Prävalenz suizidalen Verhaltens war doppelt so hoch ( $25 \%$ vs. $11 \%$ ) und knapp über die Hälfte aller Patienten mit Alkoholabhängigkeit und ADHS erfüllten die Kriterien einer antisozialen Persönlichkeitsstörung. In einer aktuellen Studie untersuchten Ohlmeier et al. (2008) 91 erwachsene Patienten mit einer Alkoholabhängigkeit und 61 Multisubstanzabhängige (Durchschnittsalter 47 Jahre, 27 \% weibliche Patienten). Bei ca. 22\% der alkoholabhängigen Patienten konnte retrospektiv der Beweis für eine ADHS Symptomatik im Kindes- und Jugendalter, die zu 33,3\% ins Erwachsenenalter persistierte, erbracht werden. Die Autoren schlussfolgern daraus, dass einer frühen adäquaten Diagnose und Therapie eine große Bedeutung zukommt. Erstaunlicherweise lag aber die Prävalenz des Alkoholkonsums in den adäquat behandelten Cruppen der MTA Studie am höchsten (Molina et al. 2007). Dennoch sollte sowohl diagnostisch als auch therapeutisch auf die erhöhte Responsivität von ADHS-Betroffenen bezüglich Suchtmittel geachtet werden, die durch die psychotrope Eigenwirkung direkt auf das Gleichgewicht des Motivationsregelkreises Einfluss haben und das Risiko für eine Abhängigkeitsentwicklung bei fehlenden regulatorischen Kompensationsmechanismen erhöhen. 


\subsection{Illegale Substanzen}

Die folgenden Betrachtungen beziehen sich nur auf eine Auswahl der gängigsten, d.h. am häufigsten konsumierten illegalen Drogen. Psychotrope Drogen dienen nicht nur der Selbstmedikation, speziell Cannabinoide und Kokain (Volkow et al. 2003), sondern deren Konsum ist auch Ausdruck riskanten Verhaltens (sensation seeking), wie es für ADHS-Betroffenen typisch ist. Eigene Untersuchungen anhand der Klientel der Rostocker Designerdrogensprechstunde (Reis und Häßler 2006) zeigen, dass die Erfassung des Suchtgeschehens im Kindes- und Jugendalters sich nicht auf eine Hauptdroge beschränken sollte, sondern auch legale Drogen und Probierkonsum umfassen, denn multiple Gebrauchsmuster sind von hoher praktischer Relevanz. Auch Wochenendkonsum birgt eine Suchtgefährdung, wenn mehrere Substanzen konsumiert werden.

\section{Cannabis}

Die folgenden Ausführungen beziehen sich ausschließlich auf den psychoaktiven Hauptbestandteil der Hanfpflanze, das Delta-9-trans-Tetrahydrocannabinol (THC) und nicht auf die rund 8o Cannabinoide, die aber auch synergistische oder antagonistische Wirkungen auf somatische und psychische Funktionen haben können.

THC besteht im Wesentlichen aus einer Benzopyran- und einer Phenolstruktur, ist fettlöslich, sammelt sich somit primär im Fettgewebe an und wird in der Leber zu wasserlöslichen nierengängigen Metaboliten umgebaut. Bei oraler Aufnahme tritt nach 30-120 min, beim Rauchen (Kiffen) bereits nach wenigen Minuten die entsprechende Wirkung ein. Während die Wirkung bei oraler Aufnahme nach ca. 3-12 h, je nach Dosis, abgeklungen ist, geschieht dies beim Rauchen bereits nach 2-3 h. Die Halbwertzeit liegt bei $52 \mathrm{~h}$, was die relativ lange Nachweisbarkeit, bis zu mehreren Wochen bei chronischem Gebrauch, erklärt. Als wirksame Dosis werden 2-20 mg beim Rauchen und $10 \mathrm{mg}$ bei oraler Zufuhr angesehen, was in 0,3-1 g Haschisch oder 1-5 g Marihuana enthalten ist (Geschwinde 1998).

Neben zwei bekannten Cannabinoidrezeptoren ( $\mathrm{CB} 1$ und $\mathrm{CB} 2$ ), die in großer Dichte in den Basalganglien, im Kleinhirn, im Hippokampus und im Frontalhirn vorkommen, besteht vermutlich eine Kolokalisation von Rezeptoren für Cannabinoide und Steroidhormone.

Die Aktivierung des Cannabinoidrezeptors hemmt einerseits das intrazelluläre Enzym Adenylatzyklase und andererseits spannungsgesteuerte $\mathrm{Ca}^{2+}-\mathrm{Ka}$ näle. Gleichzeitig wird die Leitfähigkeit bestimmter $\mathrm{K}^{+}$-Ionenkanäle erhöht. Somit kommt es zu einer Erniedrigung der Transmitterfreisetzung, indirekt auch der dopaminergen $\mathrm{D}_{1}$ - und $\mathrm{D}_{2}$-Rezeptor-mediierten Effekte und zu einer Abnahme der neuronalen Erregbarkeit, darunter einer Hemmung glutamaterger synaptischer Übertragungsvorgänge (Krausz und Lambert 200o). 


\section{Ecstasy}

Ecstasy ist ein Sammelbegriff für verschiedene Substanzen, die auf den Prototyp, das Amphetamin, zurückgeführt werden. Die Methylendioxyamphetamine, wozu als wichtigste Vertreter Methyldioxymethamphetamin (MDMA), Methyldioxyethylamphetamin (MDEA) und Methoxymethylendioxyamphetamin (MMDA) zählen, besitzen vorwiegend entaktogene Wirkungen (Herstellen einer inneren Berührung), während die methoxylierten Phenylalkanamine, deren Stammsubstanz das Meskalin ist, halluzinogene Effekte aufweisen.

Bei oraler Aufnahme setzt die Wirkung nach 20-60 min ein und hält $2 \mathrm{~h}$ (Amphetamin) bis $12 \mathrm{~h}$ (Methylendioxyamphetamin) an. Die sympathikomimetischen Effekte halten am längsten an. Durch fehlende Hydroxylgruppen am aromatischen Ring oder der Seitenkette erhöht sich die Lipophilie und damit die Penetration durch die Blut-Hirn-Schranke. Die Metabolisierung erfolgt in der Leber. Teilweise kommt es zur unveränderten renalen Ausscheidung. Während Amphetamin und Metamphetamin indirekte Noradrenalin- und Dopaminagonisten sind und somit zu einer erhöhten Freisetzung und Wiederaufnahmehemmung dieser Transmitter führen, beeinflussen Entaktogene wie MDMA eher das serotonerge System, indem sie die Serotoninkonzentration im synaptischen Spalt serotonerger Neurone steigern. Auch halluzinogene Phenylalkanamine wirken als Agonisten auf das serotonerge System, insbesondere auf den $5-\mathrm{HT}_{2 \mathrm{~A}}$-Rezeptor (Thomasius et al. 1997). Amphetamine führen zu einer größeren Leistungssteigerung als MDMA. MDMA hat dagegen eine vorrangige Wirkung auf das interpersonale Erleben und die Introspektionsfähigkeit. Halluzinatorische Effekte fehlen bei reinen Entaktogenen. Die Selbstkontrolle bleibt in der Regel erhalten. Halluzinogene Phenylalkanamine führen dagegen zu Depersonalisations- und Derealisationsphänomenen. Es kann zu einem entfremdeten Körper- und Ich-Erleben kommen. Die Selbstkontrolle geht verloren.

\section{Kokain}

Kokain, ein Alkaloid des Kokastrauches (Benzoylecgoninmethylester), kann oral, nasal, intravenös oder durch Inhalation konsumiert werden. Beim Rauchen setzt durch die vollständige Resorption über die Lunge die Wirkung schlagartig ein und hält nur kurz, d. h. 5-10 min an (Preuß et al. 200o). Bei oraler Gabe oder beim Schnupfen verläuft die Resorption über 30-6o min. Kokain wird durch hydrolytische Spaltung fast vollständig in der Leber und im Plasma metabolisiert. Die Plasmahalbwertzeit beträgt 30-90 min. Kokain setzt vermehrt Dopamin frei und hemmt bzw. behindert gleichzeitig die Wiederaufnahme mehrerer Neurotransmitter wie Dopamin, Serotonin und Noradrenalin. Es fungiert als Dopaminagonist, vorrangig der $\mathrm{D}_{1}-$ und $\mathrm{D}_{2}$-Rezeptoren . Die agonistische Wirkung wird durch ein Serotonindefizit verstärkt (Woolverton u. Johnson 1992). 


\section{Heroin}

Die verschiedenen Opiatagonisten, zu denen auch therapeutisch eingesetzte wie Morphin- $\mathrm{HCl}$, Hydromorphin, Levo-Methadon, Piritramid, Tilidin und Tramadol zählen, unterscheiden sich durch die Art ihrer Aufnahme und ihrer Metabolisierung. Im Gegensatz zu synthetischen Opiatagonisten wird Morphin nach rascher Aufnahme im Magen-Darm-Trakt bereits in der Mukosa und in der Leber konjugiert, bevor es über die Niere ausgeschieden wird. Der analgetische Wirkeffekt tritt bei i.v.-Applikation nach rund 30-40 min ein und hält 3-5 h an. Die Resorptionshalbwertzeit bei oraler Gabe liegt bei 4-6o min (Zieglgänsberger und Höllt 200o). Heroin wird erst aktiv, nachdem es zu Morphin metabolisiert wurde. Es besitzt eine höhere Lipophilie als Morphin und damit eine höhere Penetration in das ZNS. Bei i. v.-Injektion tritt der euphorisierende Effekt („rush“) unmittelbar ein, während es beim Rauchen oder Schnupfen erst nach 5-10 Minuten dazu kommt. Opiatagonisten binden an spezifische Rezeptoren mit 7 Transmembranregionen, von denen bis dato 4 Typen unterschieden werden- $\mu$-, $\delta$-, $\kappa$ - und Orphan-(ORL-1-)Rezeptoren. Für die euphorisierende Wirkung sind nur $\mu$ - und $\delta$-Agonisten verantwortlich. Opiatagonisten haben eine Reduktion der neuronalen Erregung zur Folge, wobei sie einerseits den transmembranen Kalziumstrom und andererseits die Freisetzung von L-Glutamat und Azetylcholin hemmen. Über den NMDA-Rezeptor verstärken Opiate die neuronale Plastizität und Lernprozesse, was unter anderem die Entwicklung und Konstanz psychischer und physischer Abhängigkeit erklären würde.

Bei Vorliegen einer ADHS zeigt sich ein klar erhöhtes Risiko (um ca. das 7-fache erhöht), einen Drogenmissbrauch/eine Drogenabhängigkeit zu entwickeln (Biederman etal. 1998). Bevorzugte Substanzen des Missbrauchs oder der Abhängigkeit sind Cannabinoide (Marihuana), Kokain und Stimulanzien (Ecstasy). Bei Patienten mit Kokainmissbrauch liegt die Erwachsenen-ADHS Prävalenz zwischen 10 und 33\% (Davids und Gastpar 2003). Ähnliche Häufigkeiten sind auch für andere Drogenabhängigkeiten anzunehmen. Unter den von Ohlmeier et al. (2008) untersuchten 61 Multisubstanzabhängigen wiesen ca. 52 \% eine ADHS Symptomatik mit einer Persistenz in das Erwachsenenalter von $65,5 \%$ auf. In zahlreichen Studien konnte gezeigt werden, dass eine frühzeitige adäquate Therapie mit Methylphenidat (MPH) im Kindesalter aber auch in der Pubertät das spätere Risiko eines Substanzmissbrauchs und damit die Entwicklung einer Abhängigkeit erheblich senkt (Ohlmeier et al. 2008). MPH reduziert bei Kokainabhängigen nicht nur das Verlangen sondern auch die Frequenz des Konsums und die Konsummengen (Schubiner et al. 2002).

\subsection{Videospiele und Internet}

Der englische Sozialpsychologe J. R. Brown wies darauf hin, dass sich von einem unbekannten, beliebig herausgegriffenen Menschen unserer modernen 
Industriegesellschaft sehr wenig über seine Vorlieben, seine Sprache, sein Einkommen und seine Religionszugehörigkeit sagen lasse, dagegen ließe sich mit hinreichender Sicherheit feststellen, dass er innerhalb eines 24-StundenRhythmus schlafe, trinke, esse, verdaue und - fernsehe (Sturm und Brown 1979). Mittlerweile ist nicht nur das Fernsehen eine Massenerscheinung, sondern es wird zunehmend durch Videospiele und Internet bei Jüngeren verdrängt. Doch quantitativ, ausgedrückt durch die tägliche Mediennutzungsdauer, hat sich bei den Mädchen und Jungen, verglichen mit denen der fünfziger Jahren in den USA kaum etwas verändert (s. Tab. 5).

Tab. 5 Durchschnittliche Mediennutzungsdauer in Minuten

\begin{tabular}{|lcc|}
\hline Schultag & $\begin{array}{c}\text { Baier et al. } 2005 n=5200 \\
\text { Häßler et al. 1993 } n=97 \\
\text { (nur Fernsehen) }\end{array}$ \\
\hline Fernsehen und Videozeit & 101 & \\
\hline Computer und Videospielzeit & 50 & 155 \\
\hline gesamt & 151 & \\
\hline Samstag & 153 & 257 \\
\hline $\begin{array}{l}\text { Fernsehen und Videozeit } \\
\text { Computer und Videospielzeit }\end{array}$ & 82 & \\
\hline gesamt & 236 & \\
\hline
\end{tabular}

Sie lag und liegt zwischen 2 und 4,5 Stunden. Über einen eigenen Fernseher, einen eigenen Computer und eine eigene Spielkonsole verfügten 2005 in Deutschland rund $1 / 3$ aller Kinder und Jugendlichen. Die Verfügbarkeit dieser Medien bei Mädchen war jeweils um $10 \%$, bei Spielkonsolen sogar um $23 \%$ geringer als bei den Jungen (Baier et al. 2005 www.ksan.de/download/abschlussbericht.pdf). Ohne Zweifel sagen Durchschnittswerte weder etwas über die Nutzungszeiten exzessiver Konsumenten noch über die konsumierten Inhalte aus. Bezüglich der Auswirkungen, im positiven wie im negativen Sinne, des Medienkonsums auf das Verhalten bzw. die Herausbildung psychischer Störungen gibt es sehr widersprüchliche Aussagen (Paik und Comstock 1994, Anderson 2004). In einer jüngst publizierten Studie zeigten Polman et al. (2008), dass nur bei Jungen aktives Spielen gewalttätiger Videospiele mit aggressivem Verhalten assoziiert war. Das Rezipieren blieb ohne entsprechende Folgen. Wenn aber eine psychische Störung vorliegt, so scheint auch der Medienkonsum verändert. Bereits 1993 konnte aber gezeigt werden, dass Kinder mit einer Störung des Sozialverhaltens am Wochenende mehr fern sahen als psychisch gesunde Kinder. In der Woche gab es keine Unterschiede in der Nutzungsdauer des Mediums Fernsehen (Häßler et al. 1993). 
Einen ähnlichen Zusammenhang konnten auch Yoo et al. (2004) zeigen. Sie untersuchten 264 Jungen und 271 Mädchen im Alter von durchschnittlich 11 Jahren und fanden, dass die Internetsüchtigen $(n=5)$ und die wahrscheinlich Internetsüchtigen $(\mathrm{n}=75)$ höhere Werte auf der Koreanischen Version der DuPaul ADHD Rating Scale (K-ARS) hatten. In dieser Untersuchung konnte ein signifikanter Zusammenhang zwischen dem Schweregrad der ADHD Symptomatik und dem Schweregrad der Internetsucht bei Kindern $(p<0.001)$ nachgewiesen werden. 32,7\% der ADHD Gruppe und 3.2\% der Kontrollgruppe erfüllten die Kriterien für eine Internetsucht. Die Autoren schlussfolgern, dass sowohl Unaufmerksamkeit als auch Hyperaktivität und Impulsivität Risikofaktoren für die Herausbildung einer Internetsucht seien. Spiele standen in der ADHD Gruppe im Vordergrund der Internetnutzung. Nahezu identische Ergebnisse stammen aus der Untersuchung von Chan und Rabinowitz (2006). Sie schlossen in ihre Studie 31 Jungen und 41 Mädchen mit einem Durchschnittsalter von 15,3 Jahren ein. Signifikante Zusammenhänge ergaben sich zwischen Videospielen im Internet oder über Spielekonsolen und der Conners-ADHD Gesamtskala $(\mathrm{p}<0.02)$ sowie reiner Unaufmerksamkeit $(\mathrm{p}<0.001)$. Die Untersuchung erbrachte aber weder eine signifikante Beziehung zwischen Videospielen und oppositionellem noch hyperaktivem Verhalten.

Internetspiele sind entsprechend des Neugierverhaltens/der Sensationsgier von ADHD Kindern in kürzester Zeit variabel wählbar, entsprechen einem multimodalen Stimulus (visuell und akustisch) und garantieren bei Erfolg eine unmittelbare Belohnung ohne größere zeitliche Verzögerung. Somit lassen sich mittels dieser (Spiel)Aktivitäten neurokognitive, soziale, interpersonelle und emotionale Defizite zeitweise kompensieren. Diese Flucht aus der realen in die virtuelle Welt birgt in der Tat ein hohes Suchtpotenzial in sich. Aber nur eine kleine Minderheit entwickelt jemals eine Sucht. Dennoch erhöhen steigende Konsumzeiten oder auch Spiele mit bestimmten Inhalten wiederum das Risiko des Schulversagens mit allen anderen negativen Konsequenzen. Dieser Teufelskreislauf bedarf einer frühzeitigen und konsequenten Unterbrechung, um massiven Fehlentwicklungen vorzubeugen. Tägliche Nutzerzeiten über 1 Stunde haben bereits negative Konsequenzen im sozialen und schulischen Bereich (Chan und Rabinowitz 2006). Eltern und Geschwister müssen sich somit permanent ihrer Modellwirkung und Vorbildfunktion bewusst sein und auffälligen Tendenzen, auch denen außerhalb der Familie in der Peer-Cruppe, entgegenwirken, da ADHS Kinder einerseits prädestinierter für erhöhten Fernseh-, Videospiele- und Internetkonsum und andererseits vulnerabler für deren negative Auswirkungen sind. Nicht ausgeschlossen ist auch, dass exzessiver Fernseh-, Videospiele- und Internetkonsum eine ADHSähnliche Symptomatik hervorrufen kann. 


\subsection{Stimulanzientherapie und Suchtgefahr}

Unter einer Therapie mit Methylphenidat (am sichersten ist eine Retardformulierung) besteht, obwohl Methylphenidat unter das Betäubungsmittelgesetz fällt, kein Suchtrisiko (Volkow und Swanson 2008). Häufig wird postuliert, dass die Methylphenidatbehandlung protektiv auf das Suchtrisiko der ADHS-Patienten wirkt. Die Auswirkungen der Methylphenidatbehandlung auf das Suchtrisiko sind aber umstritten (vgl. Molina 2007), da die Studien, die den Einfluss der Methylphenidatbehandlung untersucht haben (u.a. Barkley et al. 2003, Biederman et al. 1997, 1999, Molina 2007) zu widersprüchlichen Ergebnissen kommen.

\section{Literatur}

American Psychiatric Association. Diagnostic and Statistical Manual of Mental Disorder. $4^{\text {th }}$ ed. (DSM-IV), Washington, DC, 1994.

Anderson CA. An update on the effects of playing violent video games. I Adolesc 2004; 27:113-122.

Baier D, Pfeiffer C, Windzio M, Rabold S. Abschlussbericht über eine repräsentative Befragung von Schülerinnen und Schülern der 4. und 9. Jahrgangsstufe, 2006 -ksan.de.

Barkley R A, Fischer M, Smallish L, Fletcher K (2003) Does the treatment of attention-deficit/hyperactivity disorder with stimulants contribute to drug use/abuse? A 13-year prospective study. Pediatrics 111: 97-109.

Barkley RA. Attention-deficit hyperactivity disorder: A handbook for diagnosis and treatment. (3 $3^{\text {rd }}$ ed.) New York: Guilford 2006.

Biederman J, Wilens T, Mick E et al. Is ADHD a risk factor for psychoactive substance use disorders? Findings from a four-year prospective follow-up study. I Am Acad Child Adolesc Psychiatry 1997; 36:21-29.

Biederman J, Wilens TE, Mick E et al. Does attention-deficit hyperactivity disorder impact the developmental course of drug and alcohol abuse and dependence? Biol Psychiatry 1998; 44:269-273.

Biederman J, Wilens T, Mick E, Spencer T, Faraone SV. Pharmacotherapy of attention-deficit/hyperactivity disorder risk for substunce use disorder. Pediatrics 1999; 104(2):e20.

Chan PA, Rabinowitz T. A cross-sectional analysis of video games and attention deficit hyperactivity disorder symptoms in adolescents. Ann Gen Psychiatry 2006, 5:16-25.

Davids E, Gastpar M. Aufmerksamkeitsdefizit-/Hyperaktivitätsstörung und Substanzmittelabhängigkeit. Psychiat Prax 2003; 30:182-186.

Dilling H, Mombour W, Schmidt MH (Hrsg.). Internationale Klassifikation psychischer Störungen. ICD-10 Kapitel V (F). Bern: Hans Huber 2005, 293-297.

Domino EF (1998) Tobacco smoking and nicotine neuropsychopharmacology: some future research directions. Neuropsychopharmacology 18:456-468.

Elkins IJ, McGue M, lacono WG. Prospective effects of attention-deficit/hyperactivity disorder, conduct disorder, and sex on adolescent substance use and abuse. Arch Gen Psychiatry 2007; 64:1145-1152.

Geschwinde T. Rauschdrogen: Marktformen und Wirkungsweisen. Springer, Berlin Heidelberg New York, 1998.

Gillberg C, Gillberg IC, Rasmussen P, Kadesjö B, Söderström H, Rastam M. Co-existing disorders in ADHD - Implications for diagnosis and intervention. Eur Child Adolesc Psychiatry 2004; 13 (Suppl 1):80-92.

Häßler F, Gierow B, Tilch P, Langemann I. Das Fernsehverhalten einer kinder-und jugendpsychiatrischen Inanspruchnahmepopulation. Pädiatr Grenzgeb 1993; 31:363-369.

Krausz M, Lambert M. Cannabis. In: Uchtenhagen A, Zieglgänsberger W (Hrsg) Suchtmedizin. Urban \& Fischer, München, 2000:77-83.

Krause KH, Dresel SH, Krause / et al. Stimulant-like action of nicotine on striatal dopamine transporter in the brain of adults with attention deficit hyperactivity disorder. Int / Neuropsychopharmacol 2002; 5:11-113. 
Kuperman S, Schlosser SS, Kramer JR et al. Developmental sequence from disruptive behavior diagnosis to adolescent alcohol dependence. Am | Psychiatry 2001; 158:2022-2026.

McGough II, Smalley SL, McCracken IT, Yang M, Del'Homme M, Lynn DE, Loo S. Psychiatric Comorbidity in adult attention deficit hyperactivity disorder: findings from multiplex families. Am I Psychiatry 2006; 162:1621-1627.

Mick E, Biederman J, Faraone SV, Sayer J, Kleinman S. Case-control study of attention of attention-deficit hyperactivity disorder and maternal smoking, alcohol use, and drug use during pregnancy. I Am Acad Child Adolesc Psychaitry 2002 b; 23:378-385.

Molina BS, Flory K, Hinshaw SP, Greiner AR, Arnold LE, Swanson JM, Hechtman L, Jensen PS,Vitiello B, Hoza B, Pelham WE, Elliott GR, Wells KC, Abikoff HB, Gibbons RD, Marcus S, Conners CK, Epstein IN, Greenhill LL, March JS, Newcorn JH, Severe JB, Wigal T (2007). Delinquent behavior and emerging substance use in the MTA at 36 months: prevalence, course, and treatment effects.) Am Acad Child Adolesc Psychiatry. 46(8):1028-40.

Molina BSG, Pelham WEJ. Childhood predictors of adolescent substance use in a longitudinal study of children with ADHD. J Abnorm Psychology 2003; 112:497-507.

Murphy KR, Barkley RA, Bush T. Young adults with attention deficit hyperactivity disorder: subtype differences in comorbidity, educational, and clinical history. I Nerv Ment Dis 2002; 190:147-157.

Ohlmeier MD, Peters K, Te Wildt BT, Zedler M, Ziegenbein M, Wiese B, Emrich HM, Schneider U. Comorbidity of alcohol and substance dependence with attention-deficit/hyperactivity disorder (ADHD). Alcohol \& Alcoholism 2008; 43:300-304.

Paik H, Comstock $\mathrm{G}$. The effects of television violence on anti-social behavior: A meta-analysis. Commun Res 1994; 21:516-546.

Pliszka S, AACAP Work Group on Quality Issues. Practice arameters for the assessment and treatment of children and adolescents with attention-deficit/hyperactivity disorder. I Am Acad Child Adolesc Psychiatry 2007; 46:894-921.

Polman H, de Castro BO, van Aken MAG. Experimental study of the differential effects of playing versus watching violent video games on children's aggressive behavior. Aggr Behav 2008; 34:256-264.

Preuß UW, Bahlmann M, Koller G, Soyka M. Die Behandlung der Kokainabhängigkeit. Fortschr Neurol Psychiatr 2000; 68:224-238.

Rommelspacher H.Alkohol. In: Uchtenhagen A, Zieglgänsberger W (Hrsg) Suchtmedizin. Urban \& Fischer, München, 2000:30-39.

Schubiner $\mathrm{H}$, Tzelepis A, Milberger $\mathrm{S}$ et al. Prevalence of attention-deficit/hyperactivity disorder and conduct disorder among substance abusers. I Clin Psychiatry 2000; 61:244-251.

Schulze U, Trott GE. Perinatale Komplikationen bei Kindern mit hyperkinetischem Syndrom. Häufigkeit und Spezifität. Pädiatr Prax 1996; 50:383-393.

Sobanski E. Psychiatric comorbidity in adults with attention-deficit/hyperactivity disorder (ADHD). Eur Arch Psychiatry Clin Neurosci 2006: 256 (Suppl 1):1/26-31.

Sprafkin J, Gadow KD, Weiss MD, Schneider I, Nolan EE. Psychiatric comorbidity in ADHD symptom subtypes in clinic and community adults. I Att Dis 2007; 11:114-124.

Sturm H, Brown JR. Wie Kinder mit dem Fernsehen umgehen. Klett-Cotta, Stuttgart, 1979.

Sullivan MA, Rudnik-Levin F. Attention deficit hyperactivity disorder and substance abuse. Diagnostic and therapeutic considerations. Ann NY Acad Sci 2001; 931:251-270.

Thomasius R, Schmolke M, Kraus D. MDMA („Ecstasy“) - Konsum -ein Überblick zu psychiatrischen und medizinischen Folgen. Fortschr Neurol Psychiatr 1997; 65:49-61.

Uchtenhagen A. Störungen durch psychotrope Substanzen: ein Überblick. In: Uchtenhagen A, Zieglgänsberger W (Hrsg) Suchtmedizin. Urban \& Fischer, München, 2000:3-7.

Volkow ND, Wang G], Ma Y et al. Expectation enhances the regional brain metabolic and the reinforcing effects of stimulants in cocaine abusers. I Neurosciences 2003; 23:11461-11468.

Volkow ND, Swanson JM. Does childhood treatment of ADHD with stimulant medication affect substance abuse in adulthood. Am / Psychiatry 2008; 165:553-555. 
Wodarz N, Laufkötter R, Lange K, Johann M. Aufmerksamkeits-Hyperaktivitätssyndrom (ADHS) bei erwachsenen Alkoholabhängigen. Nervenheilkunde 2004; 23:527-532.

Woolverton WL, Johnson KM. Neurobiology of cocaine abuse. TIPS 1992; 13:192-200.

Yoo HJ, Cho SC, Ha I, Yune SK, Kim SI, Hwang I, Chung A, Sung YH, Lyoo IK. Attention deficit hyperactivity symptoms and Internet addiction. Psychiatry Clin Neurosci 2004; 58:487-494.

Zieglgänsberger W, Höllt V. Opiate und Opioide. In: Uchtenhagen A, Zieglgänsberger W (Hrsg) Suchtmedizin. Urban \& Fischer, München, 2000:87-97. 